\title{
Ocorrência e Antropofilia de Simulídeos em Comunidades Ribeirinhas de Rondônia, Brasil
}

\author{
Suzane Neves Velasques ${ }^{1}$, Jansen Fernandes Medeiros ${ }^{2}$, Felipe Arley Costa Pessoa ${ }^{3}$, Genimar Rebouças \\ Julião $^{3}$, Crisvaldo Cassio Silva de Souza' ${ }^{1}$ \& Luís Marcelo Aranha Camargo' ${ }^{1}$
}

1. Instituto de Ciências Biomédicas 5, Universidade de São Paulo, Monte Negro, Rondônia, Brasil e-mail: suh nevel@yahoo.com.br , crisvaldocassio@hotmail.com, spider@icbusp.org. 2. Universidade do Estado do Amazonas, Amazonas, Brasil, e-mail: jmedeiro@gmail.com (Autor para correspondência ${ }^{\varpi}$ ). 3. Centro de Pesquisas Leônidas e Maria Deane, Fundação Oswaldo Cruz, Manaus, Amazonas, Brasil, e-mail: facpessoa@gmail.com, grjuliao@gmail.com.

EntomoBrasilis 5 (1): 59-64 (2012)

\begin{abstract}
Resumo. A família Simuliidae é implicada como transmissores das filárias Onchocerca volvulus (Leuckart) e Mansonella ozzardi (Manson) na região norte do Brasil. Os objetivos do presente estudo foram (i) conhecer a fauna de simulídeos em duas comunidades ribeirinhas de Rondônia, (ii) avaliar a abundância e distribuição temporal (mensal e diária) de duas espécies de simulídeos e (iii) avaliar os possíveis efeitos da fatores climáticos na abundância dos simulídeos. As coletas dos simulídeos adultos foram realizadas entre setembro/2008 e dezembro/2009, das 08:00 às 10:00 e das 15:00 às 17:00. A espécie Cerqueirellum pydanieli Pessoa, Medeiros \& Barbosa foi a mais abundante em todos os meses e locais. Foi coletado um maior número de indivíduos no período matutino para ambas as espécies (C. pydanieli e Chirostilbia pertinax Kollar) observando-se tendências de alteração nos picos de atividade diária em função da disponibilidade de chuvas. Nessas comunidades foram coletadas 11 espécies de Simuliidae, sendo constatada pela primeira vez, a ocorrência de C. pydanieli, Ectemnaspis rorotaense (Floch \& Abonnenc) e Coscaroniellum ulyssesi (Py-Daniel \& Coscarón) no estado de Rondônia.
\end{abstract}

Palavras-chave: Amazônia; Atividade Hematofágica; Rondônia; Simuliidae.

\section{Occurrence and Antrhopophily of Simuliid Species in Riverine Communities from Rondônia State, Brazil}

Abstract. The family Simuliidae is implicated as containing the vectors of human filariid worms (Onchocerca volvulus Leuckart e Mansonella ozzardi Manson) in north region of Brazil. The objectives of this study were (i) to describe the occurrence of black fly species in two riverine communities from Rondônia; (ii) to evaluate the abundance patterns and temporal distribution (monthly and daily) of two blackfly species and (iii) to investigate possible climatic effects in the blackflies abundance. Adult collections were done during September/2008 to December/2009, in the diurnal period, 08:00 to 10:00 AM and 03:00 to 05:00 PM. Cerqueirellum pydanieli Pessoa, Medeiros \& Barbosa was the most abundant species during all months, and in both riverine communities. More blackflies were sampled during the morning than afternoon for both species (C. pydanieli and Chirostilbia pertinax Kollar). It was observed a tendency to alteration in the daily activity peaks due to the rainfall period. In these communities were captured 11 Simuliidae species, which provide new records for the state of Rondônia: C. pydanieli, Coscaroniellum ulyssesi (Py-Daniel \& Coscarón) and Ectemnaspis rorotaense (Floch \& Abonnenc).

Keywords: Amazônia; Haematophagic Activity; Rondônia; Simuliidae.

$\mathbf{O}$ s simulídeos (Diptera: Culicomorpha), popularmente chamados de piuns ou borrachudos, estão entre os hematófagos mais incômodos ao ser humano e outros animais, sendo vetores de diversos agentes etiológicos. Possuem ampla distribuição mundial, com mais de 2.060 espécies descritas (AdLer \& Crosskey 2009). Devido à importância médica-veterinária, têm sido desenvolvidos vários estudos com essa família na Amazônia. Entretanto em Rondônia, os dados sobre simulídeos estão restritos a descrição de novas espécies e registros pontuais, existindo uma lacuna no conhecimento da simulidofauna do estado. E ainda, muitas comunidades ribeirinhas ao longo dos rios Madeira, Preto e Machado são circunvizinhas às áreas endêmicas de Mansonella ozzardi (Manson) no Amazonas (Mederros et al. 2008), cujo os vetores são os simulídeos.

As espécies Cerqueirellum pydanieli Pessoa, Medeiros \& Barbosa e Chirostilbia pertinax (Kollar) são antropófilas e ambas têm importância médica. A primeira espécie é vetora potencial da filária M. ozzardi (PEssoA et al. 2008) enquanto a segunda parece estar associada à ocorrência do fogo selvagem, doença crônica e endêmica resultante de reações imunológicas severas (DELMONTES et al. 2001; Eaton et al. 1998). Portanto, o conhecimento de padrões temporais de abundância juntamente com dados da ocorrência de outras espécies podem fornecer subsídios para propostas de controle biológico e para vigilância entomológica na região. Assim, os objetivos deste estudo foram: (i) identificar a fauna de simulídeos nas comunidades de Tabajara e Dois de Novembro; (ii) comparar a abundância das espécies $C$. pydanieli e C. pertinax entre o período matutino e vespertino, entre as localidades e estações e (iii) avaliar os efeitos da temperatura, umidade relativa do ar, pluviosidade e pressão atmosférica na abundância das duas espécies.

\section{MATERIAL E MÉTODOS}

O estudo foi realizado em duas comunidades ribeirinhas do município de Machadinho D'Oeste, estado de Rondônia. As comunidades Tabajara (S o8 ${ }^{\circ} 56^{\prime} 38,50^{\prime \prime}$; W $62^{\circ}$ o3' $31,10^{\prime \prime}$ ) e Dois de Novembro (S $08^{\circ} 54^{\prime}$ oo, $49^{\prime \prime}$; W $62^{\circ} 11^{\prime} 32,79^{\prime \prime}$ ) situamse na região noroeste de Rondônia, às margens do rio Machado (Figura 1), e distam cerca de $700 \mathrm{~km}$ da capital Porto Velho. A atividade pesqueira em pequena escala é a principal fonte de renda dos moradores dessas comunidades, que possuem cerca 


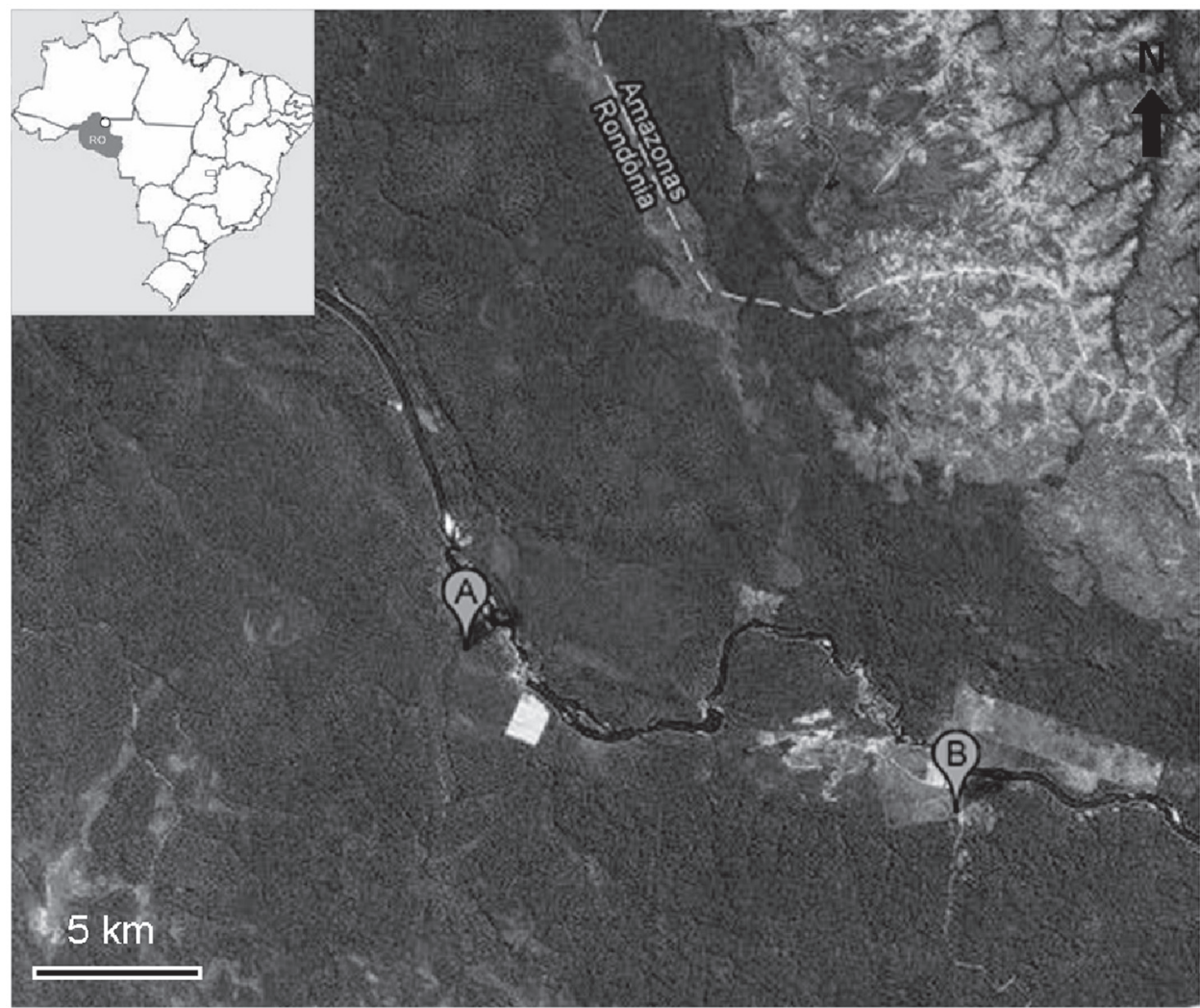

Figura 1. Localização dos pontos de coleta de simulídeos, nas comunidades Dois de Novembro (A) e Tabajara (B), às margens do rio Machado, Roraima, Brasil (Fonte: Google Maps, http://maps.google.com.br).

de 330 habitantes em Tabajara e 25 em Dois de Novembro.

Foram realizadas oito expedições de quatro dias a cada dois meses de setembro/2008 a dezembro/2009, e as coletas foram feitas das 08:00 às 10:00 e das 15:00 às 17:00. O critério de escolha dos horários baseou-se nos picos de abundância observados por Medeiros \& Py-Daniel (2003a; 2003b) e Medeiros et al. (2006).

Os simulídeos adultos foram coletados com capturadores de sucção durante o pouso em humanos; os imaturos (larvas e pupas), em substratos rochosos, folhas, galhos caídos e macrófitas com auxílio de pinças no rio Machado e igarapés da região. Adultos e imaturos foram acondicionados em frascos com álcool $70 \%$. No laboratório foram identificados a partir das características morfológicas de adultos, larvas e pupas, segundo a chave de Coscarón \& Coscarón-Arias (2007). A nomenclatura de Py-DANiEL \& Moreira-SAMPAIO (1995) foi adotada para identificação genérica. Preferimos manter a espécie $C$. pydanieli como válida, embora tenha sido sinonimizada como variação morfológica por SHELLEY et al. (2010) enquanto não forem apresentados argumentos mais sólidos, como também estudos moleculares ou citotaxonômicos.

Os parâmetros climáticos, pluviosidade mensal (mm) e pressão atmosférica diária $(\mathrm{hPa})$ foram obtidos no banco de dados do Instituto de Pesquisas Espaciais (INPE 2010). A temperatura $\left({ }^{\circ} \mathrm{C}\right)$ e umidade relativa do ar (\%) foram medidas no local de coleta com termo-higrômetro digital (marca Alla France). Foram realizadas médias mensais dos valores horários da pressão atmosférica $(\mathrm{hPa})$, temperatura e umidade relativa para avaliar o efeito dessas variáveis na abundância mensal de simulídeos. Já a pluviosidade mensal consistiu do somatório dos valores diários em milímetros (mm)

A distribuição dos dados foi previamente testada com relação a sua normalidade através do teste Kolmogorov-Smirnov; como as variáveis número de simulídeos adultos, temperatura, umidade relativa do ar e pressão atmosférica não apresentaram distribuição normal, optou-se por testes não paramétricos. Para cada espécie, o número de indivíduos coletados no período matutino e vespertino foi comparado por meio do teste de aderência qui-quadrado $\left(\chi^{2}\right)$ para várias amostras, com nível de decisão em $5 \%(\alpha=0,05)$. O teste de Mann-Whitney (U) foi adotado na comparação do número de simulídeos, de cada espécie, entre as duas localidades (Tabajara e Dois de Novembro). Para avaliar o efeito da sazonalidade na abundância de simulídeos foram criadas três classes de estação: chuvosa 1 (dezembro de 2008, janeiro e dezembro de 2009), chuvosa 2 (março e maio/2009) e seca (setembro de 2008 e julho e novembro de 2009), comparadas através do teste de Kruskal-Wallis (H). O coeficiente de correlação não paramétrico de Spearman foi usado para testar a associação entre a abundância de simulídeos (com transformação para raiz quadrada de $n+0,5)$ e os fatores climáticos. Para melhor visualização das comparações locais e temporais de abundância das duas espécies, utilizou-se a raiz quadrada (RQ) do número de indivíduos $(\mathrm{n}+0,5)$ nos gráficos.

\section{RESULTADOS E DISCUSSÃO}

Nas comunidades de Tabajara e Dois de Novembro foram capturados 1.912 indivíduos de setembro de 2008 a dezembro de 2009, com maior abundância da espécie $C$. pydanieli $(\mathrm{n}=1.813$; 94,5\%) em relação à C. pertinax (n=99; 5,5\%).

Em relação ao número de indivíduos coletados entre os períodos matutinos e vespertinos o teste qui-quadrado indicou que as amostras são homogêneas uma vez que os valores observados e esperados variaram na mesma direção, ou seja, um número significativamente maior de simulídeos foi coletado no período matutino (C. pertinax - $\chi^{2}=15,36$; C. pydanieli $-\chi^{2}=66,41$; $\mathrm{p}<0.0001)$. No sul da Amazônia venezuelana e em Caracaraí (Roraima) C. oyapockense (Floch \& Abonnenc) demonstrou atividade hematofágica ao longo de todo o dia (GRILLET et al. 2001; Ríos-Velásquez \& LuZ 2003), apresentando em algumas situações, pico bimodal (Grillet et al. 2001). No entanto, Medeiros et al. (2006) destacam que os picos de atividade diária podem sofrer mudanças ao longo das estações do ano: na época de chuvas, $C$. argentiscutum (Shelley \& Luna Dias) apresentou picos de atividade no período vespertino, enquanto picos de atividade matutinos foram observados na estação seca. Em relação à espécie $C$. pertinax (Figura 2a), nenhuma tendência foi detectada, possivelmente relacionado ao baixo número de indivíduos capturados. No presente estudo, observou-se um 
A) C. pertinax

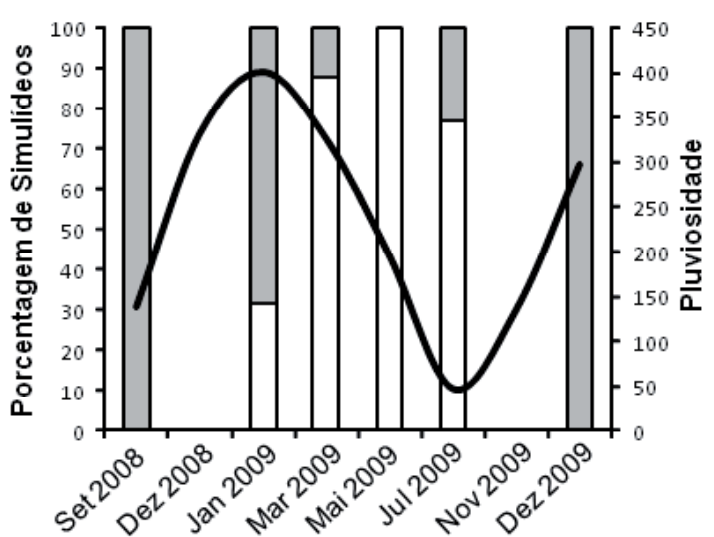

B) C. pydanieli

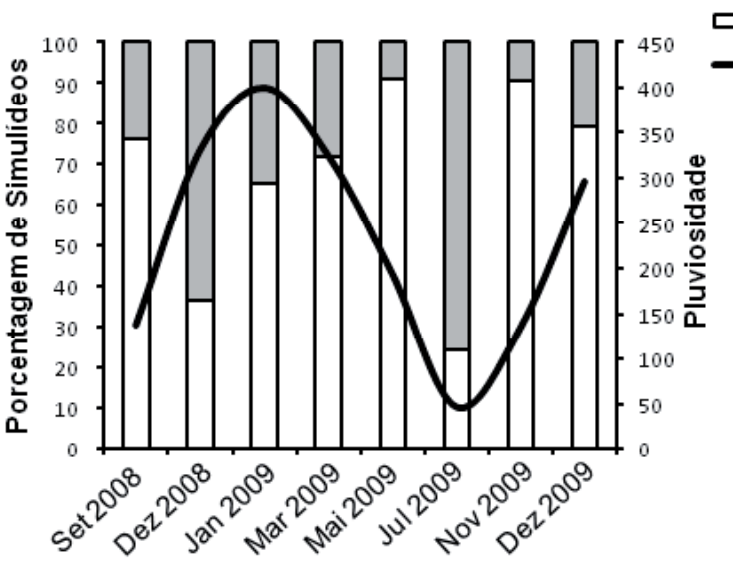

$\square$ Tarde

Manhã Pluviosidade (mm)

Figura 2. Comparação da porcentagem de simulídeos entre o período matutino e vespertino, em função da disponibilidade de chuvas durante o período de setembro de 2008 a dezembro de 2009, nas comunidades de Tabajara e Dois de Novembro, RO, Brasil.

padrão inverso, no qual a espécie $C$. pydanieli (Figura $2 \mathrm{~b}$ ) tende a ser mais ativa no período matutino após o início da estação chuvosa, enquanto picos de atividade vespertina são verificados durante os períodos mais secos.

Na comunidade de Tabajara foi coletado um maior número de simulídeos, (Figura 3). As duas comunidades "pesqueiras" diferiram significativamente quanto ao número de indivíduos de C. pertinax capturados $(\mathrm{U}=470,00 ; \mathrm{p}=0,006)$; sendo observado o mesmo padrão para C. pydanieli, apesar da não significância do teste de Mann-Whitney ( $U=401,00 ; p=0,294)$.

Os fatores climáticos pressão atmosférica, temperatura, umidade e pluviosidade não apresentaram correlações significativas com o número de simulídeos coletados $\left(\mathrm{r}_{\mathrm{s}}=-0,476, \mathrm{p}=0,233 ; \mathrm{r}_{\mathrm{s}}=\right.$ $0,476, p=0,233 ; r_{s}=-0,373, p=0,362 ; r_{s}=0,1434, p=0,4940$, respectivamente). No entanto, uma relação entre pressão atmosférica, temperatura e umidade e número de simulídeos coletados foi observada no estado do Rio Grande do Sul por Strieder \& Corseuil (1992) e por Medeiros \& Py-Daniel (1999) em Roraima, mostrando que essas variáveis afetam a atividade diária dos simulídeos. LACEY \& CHARLWOOD (1980) observaram maior intensidade de atividade hematofágica de Cerqueirellum sp. à medida que a temperatura e luminosidade aumentavam; já a diminuição da umidade relativa resultou em redução na atividade hematofágica.

Apesar do número de simulídeos coletados não apresentar correlação com a variação na pluviosidade mensal, observouse uma tendência de aumento na quantidade de indivíduos capturados nos meses de maior pluviosidade: dezembro/o8, janeiro/o9 e março/o9 (Figura 4, Tabela 1). Similarmente, não foram observadas diferenças significativas na comparação da abundância de simulídeos entre as classes de estações ( $C$. pertinax: $\mathrm{H}=0,861 \mathrm{p}=0,650 ;$ C. pydanieli: $\mathrm{H}=2,411 \mathrm{p}=0,300$ ), embora tenha sido coletado um maior número de indivíduos de C. pydanieli no início da estação chuvosa (EC1) e de C. pertinax no final desta mesma estação (EC2) (Figura 5). A relação direta e positiva entre a quantidade de chuvas e a abundância de simulídeos tem sido reportada em alguns estudos (Medeiros \& PyDANIEL 1999; Py-DANiel et al. 2000). O aumento da precipitação pode ser considerado fator determinante no estabelecimento de criadouros para simulídeos imaturos (larvas e pupas) e por conseqüência responsável pelo aumento da população de adultos (Py-DANIEL \& RAPP PY-DANiEl 1998; GRILlet et al. 2001). As espécies de simulídeos enfocadas no presente estudo, possivelmente são afetadas pelo regime de chuvas: $C$. pydanieli tem oviposição associada a rios de grandes vazões, enquanto $C$. pertinax, que

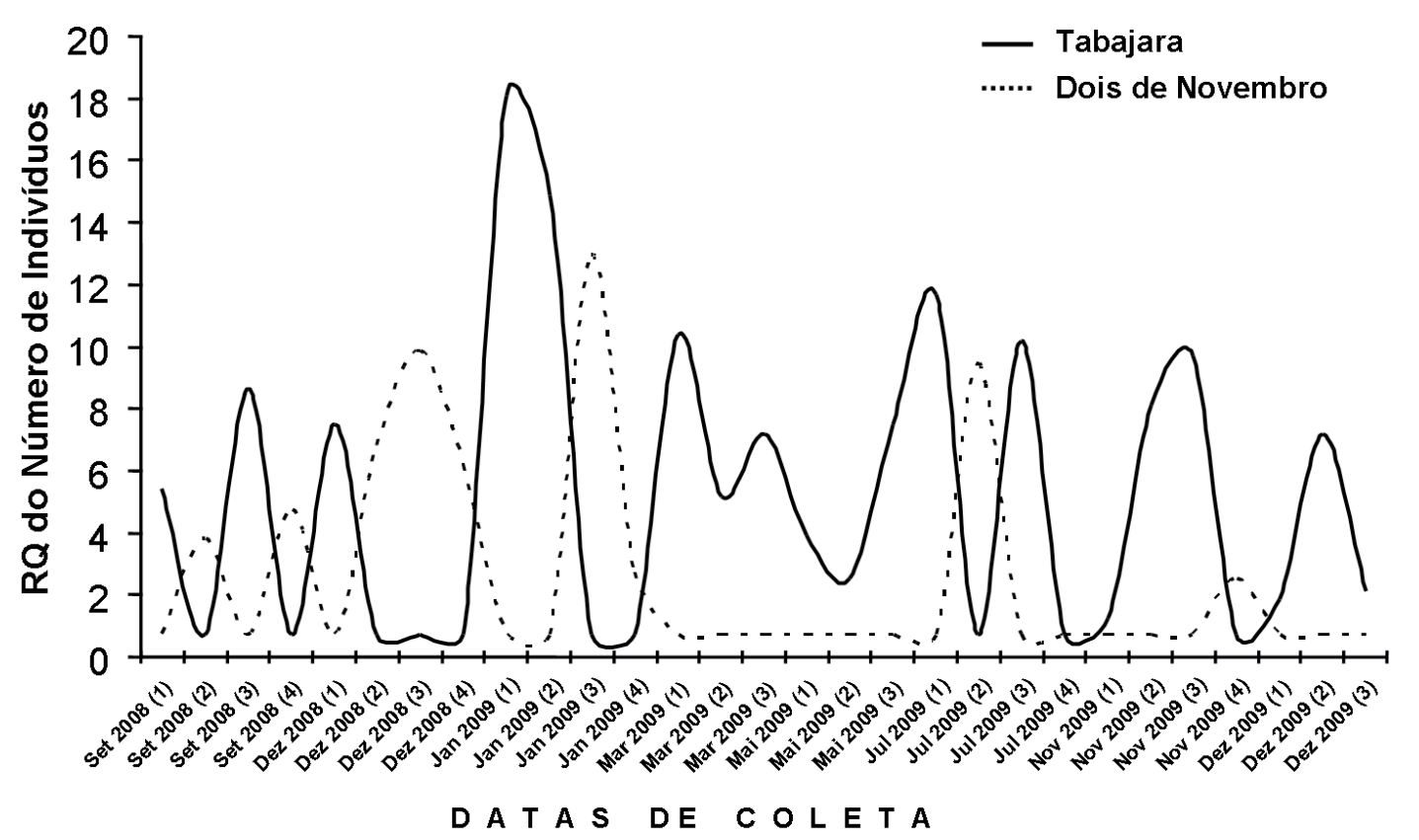

Figura 3. Comparação da abundância de simulídeos (raiz quadrada do número de indivíduos) entre as comunidades Tabajara e Dois de Novembro, RO, Brasil, amostradas durante o período de setembro de 2008 a dezembro de 2009. 
Tabela 1. Número de simulídeos coletados e as variáveis climáticas registradas durante período de setembro de 2008 a dezembro de 2009 , nas comunidades de Tabajara e Dois de Novembro, estado de Rondônia.

\begin{tabular}{|c|c|c|c|c|c|c|}
\hline $\begin{array}{l}\text { Período da } \\
\text { coleta }\end{array}$ & C.pertinax & C. pydanieli & $\begin{array}{c}\text { Umidade } \\
\text { Relativa (\%) * }\end{array}$ & $\begin{array}{c}\text { Temperatura } \\
\left({ }^{\circ} \mathbf{C}\right)^{*}\end{array}$ & $\begin{array}{c}\text { Pressão } \\
\text { Atmosférica } \\
(\mathrm{hPa})^{*}\end{array}$ & $\begin{array}{l}\text { Pluviosidade } \\
\quad(\mathbf{m m}) \dagger\end{array}$ \\
\hline Set/o8 & 1 & 138 & 68,8 & 35,3 & 28,4 & 139 \\
\hline Dez/o8 & o & 237 & 80 & 29,4 & 28,1 & 332,4 \\
\hline Jan/o9 & 19 & 704 & 73,1 & 30 & 28,7 & 401,5 \\
\hline Mar/o9 & 56 & 128 & 75,6 & 27,4 & 28,7 & 326,4 \\
\hline Mai/o9 & 1 & 76 & 87,9 & 28,5 & 28,4 & 195,5 \\
\hline Jul/o9 & 17 & 311 & 56,6 & 33,9 & 28,5 & 47,7 \\
\hline Nov/o9 & o & 166 & 55,4 & 28,3 & 28,7 & 136 \\
\hline Dez/o9 & 5 & 53 & 93,8 & 25,1 & 29,2 & 297,8 \\
\hline
\end{tabular}

* Médias mensais; † Somatório mensal

tem preferência por pequenos córregos e igarapés (CROsskeY 1993; Ruas-Neto \& Matias 1985; Vulcano 1981).

Em relação aos imaturos, foram coletadas 11 espécies: Kempfsimulium simplicicolor (Lutz), Inaequalium subnigrum (Lutz), Ectemnaspis rorotaense (Floch \& Abonnenc), E. perflava (Roubaud), Coscaroniellum quadrifidum (Lutz), C. ulyssesi (Py-Daniel \& Coscarón), C. goeldii (Cerqueira \& Nunes de Mello), C. amazonicum (Goeldi), C. pydanieli, C. pertinax e Psaroniocompsa minuscula (Lutz). Destas, $K$. simplicicolor, $C$. amazonicum, $C$. argentiscutum, $C$. pertinax e $P$. minuscula são antropófilos. São feitas três novas ocorrências: E. rorotaense, $C$. ulyssesi e C. pydanieli.

Neste estudo constatou-se, pela primeira vez, a ocorrência de $C$. pydanieli fora do estado do Amazonas, com os primeiros registros da biologia dessa espécie. O maior número de simulídeos na comunidade Tabajara, fato provavelmente relacionado à maior concentração humana combinada a grande disponibilidade de criadouros, como a área marginal à parte plana do Rio Machado, onde existem pequenos igarapés de entorno, dois habitats propícios para ambas as espécies. Com as novas ocorrências de simulídeos, aumentando o registro da fauna para 11 espécies, aproxima-se com o esperado de diversidade para a região amazônica. Nos arredores da cidade de Manaus, estado do Amazonas, 12 espécies foram registradas (HAMADA \& ADLER 2001).

Alguns estudos constataram os efeitos de variáveis climáticas como umidade relativa do ar, temperatura e pressão atmosférica na ocorrência e atividade hematofágica de piuns (MedeIros et al. 2006; ANdreazze et al. 2002; Moreira et al. 1994). Nas comunidades de Tabajara e Dois de Novembro esses fatores não afetaram significativamente as capturas dos simulídeos. Tal fato poderia ser explicado pelo número de amostras tomadas, tanto no tempo como no espaço. Dessa forma, fica evidente a necessidade de estudos com longa duração na região norte do Brasil, que ampliem o número de pontos amostrais e visem à coleta de formas adultas e imaturas, esclarecendo assim aspectos básicos da ecologia e biologia deste grupo.

A compreensão dos padrões de picada e picos de atividade estacional dos simulídeos é de suma importância, pois através da atividade hematofágica, esses insetos interferem na qualidade de vida das pessoas, causam prejuízos sanitários e até mesmo econômicos (Ruas-Neto \& Matias 1985, Strieder \& Corseuil 1992). Além disso, populações de simulídeos antropófilas têm aumentado em conseqüência das interferências antrópicas no ambiente (Dellome Filho 1991, 1992; Machado 2009). Desse modo, a investigação sobre simulídeos hematófagos em

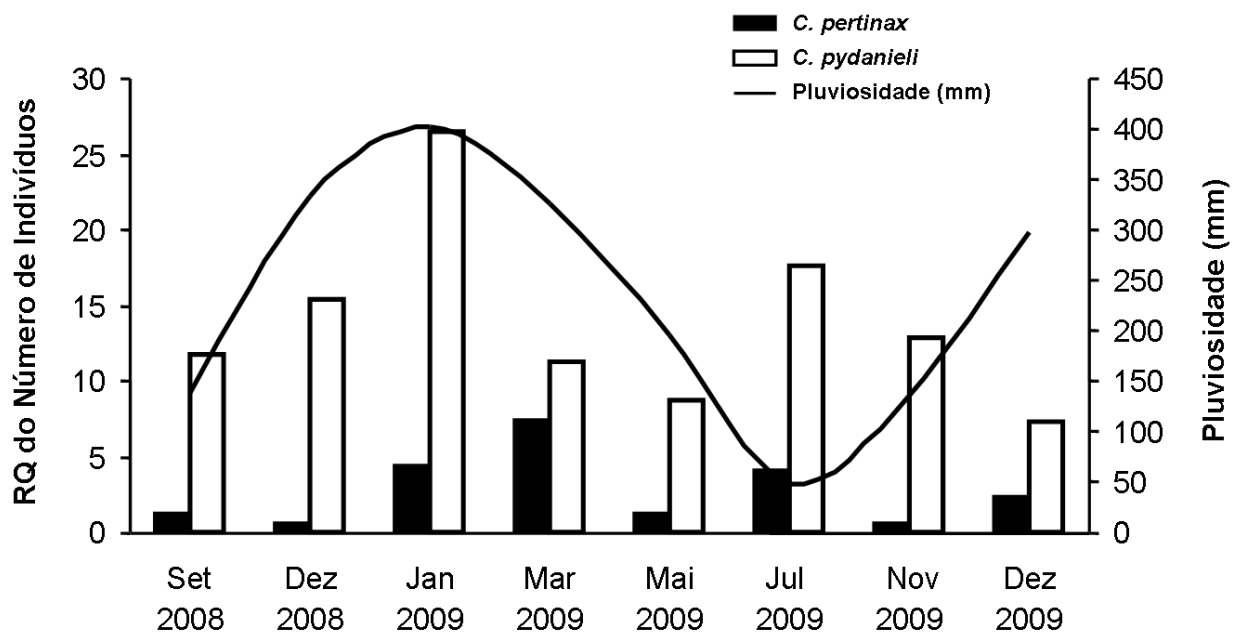

MES E S DE C O L E T A

Figura 4. Relação entre pluviosidade mensal $(\mathrm{mm})$ e o número de simulídeos (raiz quadrada do número de indivíduos) coletados em Tabajara e Dois de Novembro, Rondônia, Brasil, no período de setembro de 2008 a dezembro de 2009. 


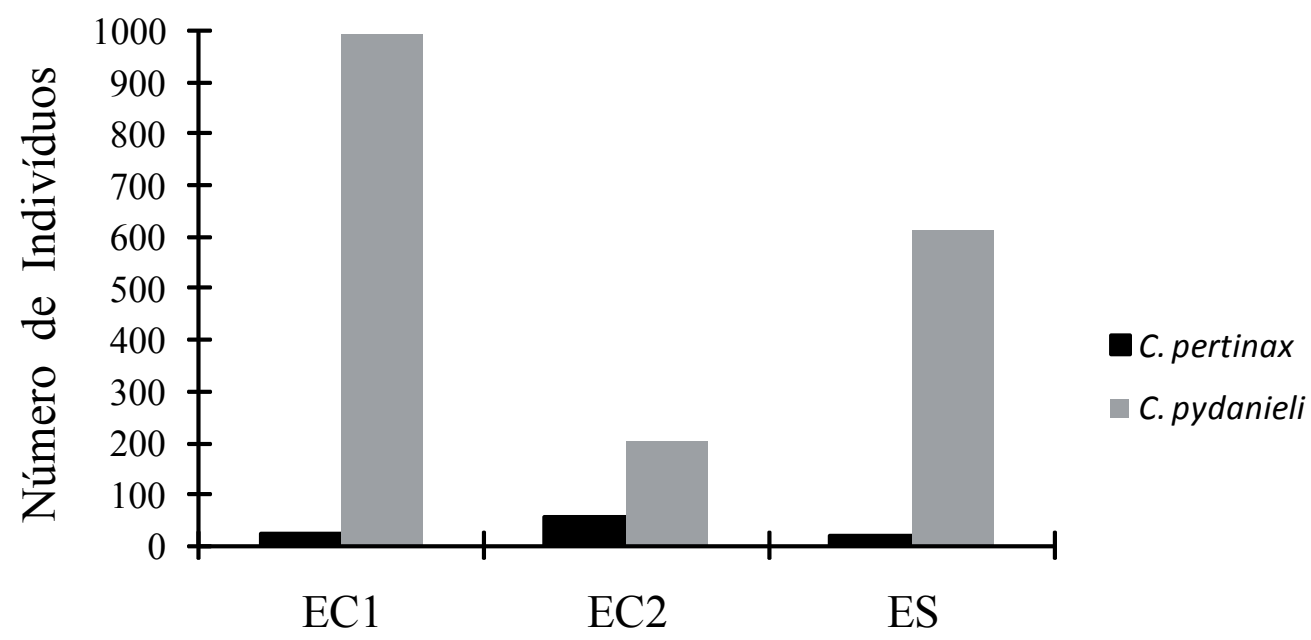

\section{Classes}

Figura 5. Número de simulídeos amostrados em três classes de estação: chuvosa 1 (EC1), chuvosa 2 (EC2) e seca (ES) nas comunidades Tabajara e Dois de Novembro, Rondônia, Brasil, no período de setembro de 2008 a dezembro de 2009.

comunidades ribeirinhas e rurais do norte do Brasil poderá fornecer subsídios para um eventual controle destes insetos, caso o incremento populacional dificulte o desenvolvimento de atividades econômicas, como a pesca esportiva, de subsistência e o ecoturismo.

\section{AGRADECIMENTOS}

A FAPESP (processo 2007/00531-5) pelo suporte financeiro para realização do projeto. Ao Dr. Victor Py-Daniel pela confirmação na identificação das espécies de simulídeos.

\section{REFERÊNCIAS}

Adler, P.H. \& R.W. Crosskey, 2009. World blackflies (Diptera: Simuliidae): A comprehensive revision of the taxonomic and geographical inventory. Disponível em: http://entweb.clemson.edu/biomia/pdfs/blackflyinventory. pdf. [Acesso em: 2010].

Andreazze, R., V. Py-Daniel, \& J.F. Medeiros. 2002. Influência dos fatores climáticos na atividade hematofágica de Psaroniocompsa incrustata (Lutz, 1910) (Diptera, Simuliidae) vetor de Onchocerca volvulus (Leuckart, 1910) em Xitei/Xidea, área indígena Yanomami, Roraima, Brasil. Entomologia y Vectores, 9: 559 - 577.

Coscarón, S. \& C.L. Coscarón-Arias, 2007. Neotropical Simuliidae (Diptera: Simuliidae). Aquatic biodiversity in Latin America (ABLA), Vol. 3. Sofia-Moscow: Pensoft, 685 p.

Crosskey, R.W, 1993. Blackflies (Simuliidae). p. 240-287 In: Lane, R.P. \& R.W. Crosskey (Eds.). Medical Insects and Arachnids. London: Chapman \& Hall.

Dellome Filho, J., 1991. Simuliofauna do Rio Morumbi, Morretes (PR). I Coleta e criação; dados meteorológicos e físicoquímicos do criadouro; adultos (Diptera, Simuliidae). Acta Biológica Paranaense, 20: 145-156.

Dellome-Filho J., 1992. Simuliofauna do Rio Morumbi, Morretes (PR). II Substratos naturais e artificiais dos imaturos e fauna associada (Diptera, Simuliidae). Acta Biológica Paranaense 21: 77-88.

Delmontes, S., J. Kanitakis, E. Cozzani, A. Parodi \& A. Rebora, 2001 . Diagnosing Pemphigus foliaceus: a retrospective analysis of clinical, histological and immunological criteria. Dermatology, 203: 289-293.

Eaton D.P., L.A. Diaz, G. Hans-Filho, V.D. Santos, V. Aoki, H. Friedman, E.A. Rivitti, S.A.P. Sampaio, M.S. Gottlieb, G.J. Giudice, A. Lopez \& E.W. Cupp, 1998. Comparison of black fly species (Diptera: Simuliidae) on an Amerindian reservation with a hight prevalence of "fogo selvagem" to neighbouring diseasefree sites in the state of Mato Grosso do Sul, Brazil The
Cooperative Group on Fogo Selvagem Research. Journal of Medical Entomology, 35:120-131.

Grillet M.E., M.G. Basáñez, S. Vivas-Martínez, N.J. Villamizar, H.L Frontado, J. Cortez, P. Coronel \& C. Botto, 2001. Human onchocerciasis in the Amazonian area of southern Venezuela: spatial and temporal variations in biting and parity rates of black fly (Diptera: Simuliidae) vectors. Journal of Medical Entomology, 38: 520-530.

Hamada, N. \& P.H. Adler, 2001. Bionomia e chave para imaturos e adultos de Simulium (Diptera: Simuliidae) na Amazônia Central, Brasil. Acta Amazônica, 31: 109-132.

INPE, 2010. Instituto Nacional de Pesquisas Espaciais. Consulta específica ao Banco de Dados por Mês/ Ano, com filtros em alguns sensores. Disponível em: http://satelite.cptec.inpe.br/PCD/historico/consulta pcdm. jsp. [Acesso em: março 2010].

Lacey, L.A. \& J.D. Charlwood, 1980. On the biting activities of some antropophilic Amazonian Simuliidae (Diptera). Bulletin of Entomological Research, 70: 495-509.

Machado, A., 2009. Os borrachudos, o turismo rural e a vida de Camboriú. Disponível em: http://aderbalmachado.blogspot. com/2009/11/os-borrachudos-o-turismo-rural-e-vida.html. [Acesso em: abril 2010]

Medeiros, J.F. \& V. Py-Daniel, 1999. Atividade hematofágica e infecção natural de três espécies de Simuliidae (Diptera: Culicomorpha) em Xitei/Xidea, área indígena Yanomami, Roraima, Brasil. Entomología y Vectores, 6: 210-226.

Medeiros, J.F. \& V. Py-Daniel, 2003a. Estado fisiológico e preferência de picadas de Cerqueirellum argentiscutum (Shelley \& Luna Dias, 1980) (Diptera: Simuliidae), vetor de Mansonella ozzardi (Manson, 1897) (Nematoda: Onchocercidae) no Brasil. Entomología y Vectores, 9: 505517.

Medeiros, J.F. \& V. Py-Daniel, 2003b. Atividade hematofágica diária e taxa de infecção de Cerqueirellum argentiscutum (Shelley \& Luna Dias) (Diptera: Simuliidae) por Mansonella ozzardi (Manson) (Nematoda: Onchocercidae) em uma comunidade do Rio Solimões, Amazonas, Brasil. Entomología y Vectores, 10: 9-20.

Medeiros, J.F., V. Py-Daniel \& T.J. Izzo, 2006. The influence of climatic parameters in the haematophagic daily activity of Cerqueirellum argentiscutum (Shelley \& Luna Dias) (Diptera: Simuliidae) in Amazonas, Brazil. Acta Amazonica, 36: $563-568$.

Medeiros, J.F., V. Py-Daniel, U.C. Barbosa \& G.M. Ogawa, 2008. Current profile of Mansonella ozzardi (Nematoda: Onchocercidae) in communities along the Ituxi river, Lábrea municipality, Amazonas, Brazil. Memórias do Instituto 
Oswaldo Cruz, 103: 409-411.

Moreira, G.R.P., R.A. Pegoraro, \& G. Sato, 1994. Influência de fatores abióticos sobre o desenvolvimento de Simulium nogueirae D'Andretta \& González em um córrego da Mata Atlântica. 1994. Anais da Sociedade Entomológica do Brasil, 23: 525-542.

Pessoa, F.A.C., U.C. Barbosa \& J.F. Medeiros, 2008. A new species of Cerqueirellum Py-Daniel, 1983. (Diptera: Simuliidae) and proven new vector of mansonelliasis from the Ituxi River, Amazon basin, Brazil. Acta Amazonica, 38: 569-578

Py-Daniel, V. \& L.H. Rapp Py-Daniel, 1998. Dinâmica de dispersão, padrões de distribuição geográfica e aspectos relativos a transmissão de Onchocerca volvulus (Leuckart, 1983) por simulídeos (Diptera: Culicomorpha, Simuliidae) na bacia hidrográfica do rio Amazonas. Entomologia Vectores, 5: 191-215.

Py-Daniel, V. \& R.T. Moreira-Sampaio, 1995. Gêneros e espécies de Simuliidae (Diptera: Culicomorpha) assinalados para o Brasil até 1995. Entomología y Vectores, 2: 117-121.

Py-Daniel, V., R. Andreazze \& J.F. Medeiros, 2000. Projeto piloto Xitei/Xidea (Roraima). I - Índices epidemiológicos de transmissão de Onchocerca volvulus (Leuckart, 1983) para os anos de 1995 - 1996. Entomología y Vectores, 7: 389-444.

Ríos-Veslásquez, C.M.\&S.L.B.Luz, 2003. Atividadehematofágica de Simulium oyapockense (Diptera: Simuliidae) na Cachoeira do Bem Querer, Caracaraí (RR). Anais do VI Congresso de Ecologia do Brasil, 44-45.

Ruas-Neto, A.L. \& R.S. Matias, 1985. Controle integrado do Simulium (Chirostilbia) pertinax Kollar, 1832. A competição interespecífica como possível método de controle natural. Boletim da Saúde, 12: 21-24.

Shelley, A.J., L.M. Hernández, M. Maia-Herzog, A.P.A. Luna Dias \& P.R. Garritano, 2010. The blackflies (Diptera: Simuliidae) of Brazil. In Arias, J.R., Golovatch, S., Wanrzen, K.M. \& E.Dominguez (Eds.): Aquatic Biodiversity in Latin American Biodiversidad Acuática en América Latina (ABLA). Vol 6. Pensoft, Sofia-Moscow, 821 pp.

Strieder, M.N. \& E. Corseuil, 1992. Atividades de hematofagia em Simuliidae (Diptera, Nematocera) na Picada Verão, Sapiranga, RS - Brasil. Acta Biologica Leopoldensia, 14: 7598.

Vulcano, M.A., 1981. Simuliidae. In: Hurlbert, S.H., G. Rodriguez \& N. Dias dos Santos (Eds.). Aquatic Biota of Tropical South America, I. Arthropoda. San Diego: San Diego State University. $323 \mathrm{p}$.

\section{Recebido em: 18/o7/2011}

Aceito em: 23/o1/2012

\section{Como citar este artigo:}

Velasques, S.N., J.F. Medeiros, F.A.C. Pessoa, G.R. Julião, C.C. S. de Souza \& L.M.A. Camargo, 2012. Ocorrência e Antropofilia de Simulídeos em Comunidades Ribeirinhas de Rondônia, Brasil. EntomoBrasilis, 5(1): 59-64.

Acessível em: http://www.periodico.ebras.bio.br/ojs/index.php/ebras/article/view/174
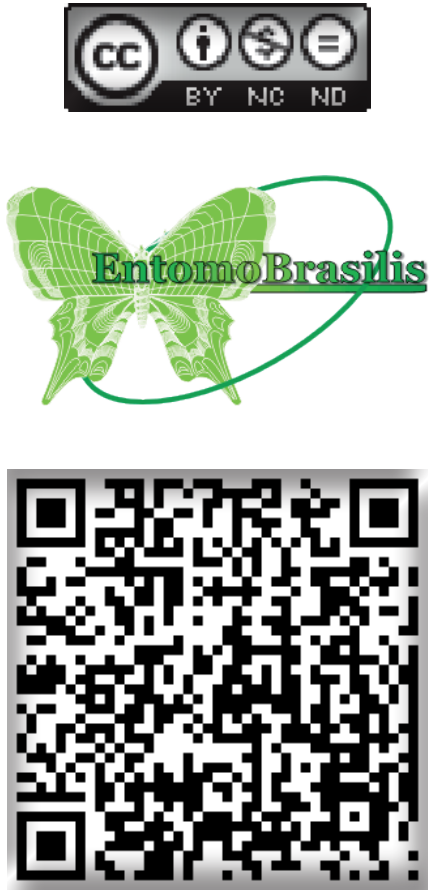